Meta

Journal des traducteurs

Translators' Journal

\title{
Paradoxes du discours québécois sur la traduction
}

\section{Sherry Simon}

Volume 35, numéro 1, mars 1990

Actes du colloque international " La traduction proligère "

URI : https://id.erudit.org/iderudit/004148ar

DOI : https://doi.org/10.7202/004148ar

Aller au sommaire du numéro

Éditeur(s)

Les Presses de l'Université de Montréal

ISSN

0026-0452 (imprimé)

1492-1421 (numérique)

Découvrir la revue

Citer cet article

Simon, S. (1990). Paradoxes du discours québécois sur la traduction. Meta,

35(1), 214-218. https://doi.org/10.7202/004148ar d'utilisation que vous pouvez consulter en ligne.

https://apropos.erudit.org/fr/usagers/politique-dutilisation/ 


\title{
PARADOXES DU DISCOURS QUÉBÉCOIS SUR LA TRADUCTION
}

\author{
SHERRY SIMON \\ Université Concordia, Montréal, Canada
}

Il n'est plus nécessaire d'insister dans une réunion de traductologues sur la pertinence du contexte social et culturel dans l'étude de la traduction. Il faut souligner toutefois qu'au cours des dernières années notre compréhension de ce que cette notion peut recouvrir a changé de façon substantielle. Du moment ponctuel de la communication qui a été identifié par les sociolinguistes, le contexte s'est élargi pour embrasser les conceptualisations et les pratiques qui accompagnent et englobent la traduction et qui soutiennent le régime particulier d'équivalence. L'émetteur et le destinataire, de simples points ou positions qu'ils étaient, se voient pourvus d'une existence sociale et historique; de laboureurs anonymes, ils se voient dotés d'un nom propre ou du moins d'une appartenance collective. Ils - et elles - se voient engagés dans des projets de communication qui comprennent des asymétries et des rapports de dominance. Ces projets de traduction, enfin, on a compris qu'il fallait les étudier de concert avec d'autres systèmes de symbolisation, d'autres pratiques d'écriture, qu'il fallait en somme les replacer dans l'histoire culturelle dont elles étaient issues.

Au Québec comme ailleurs, la traduction intervient dans une pluralité de contextes d'ordre différent. Ces contextes, on commence tout juste à les discerner de façon rigoureuse, tantôt en engageant des travaux historiques, tantôt en isolant certaines aires discursives comme celle qu'a étudiée par exemple Annie Brisset dans le domaine théâtral, où les principes de la traduction participent à la même saturation idéologique dont la langue s'investit dans plusieurs sphères de l'écriture (Brisset, à paraître). En dehors de ces contextes discursifs spécifiques, cependant, on peut parler d'une certaine toile de fond, un discours très général sur la traduction au Québec, qui accompagne la question de la langue depuis les débuts ${ }^{1}$ et qui dessine un horizon relativement immobile. On trouve tout au long des années les mêmes termes, langue, culture, traduction, mais qui entrent dans des configurations différentes. Je voudrais essayer maintenant de comprendre les sens, voire les paradoxes que ces mots recouvrent dans le but tout particulièrement de suivre le rapport entre langue et culture. Il devient évident qu'au-delà d'une certaine vérité de La Palice qui consiste à dire que la langue et la culture ont partie liée, on en vient à opposer ces deux termes et en faire le lieu de certaines valorisations implicites. Où poser la frontière entre langue et culture? La question suit et nargue le discours sur la traduction au Québec depuis toujours.

J'entrerai dans mon propos par le biais d'un personnage de roman. Les Grandes Marées de Jacques Poulin, publié en 1978 et considéré l'annonciateur du postmoderne au Québec, a pour héros un traducteur de bandes dessinées qui s'appelle TDB ou Teddy. Il habite seul sur une île dans le Saint-Laurent et son éditeur lui envoie régulièrement des bandes à traduire. Teddy a tous les tics du traducteur: il est solitaire, obsédé par le langage, attentif aux moindres nuances des mots, précis, consciencieux et ordonné dans ses habitudes de travail, friand de dictionnaires. Il n'est ni aventurier, ni voyageur; il n'est même pas très curieux. Peu communicatif, il n'est pas très doué pour les rapports interpersonnels. À part ses dictionnaires, son compagnon préféré est une machine lance-balles 
avec laquelle il joue au tennis. Ses rapports avec cette machine qu'il appelle Prince sont empreints de la même courtoisie et dignité que ses rapports avec le langage. Chaque geste est un rituel commandé par une éthique de respect. Nous apprendrons cependant avec Teddy, à la fin, que son patron avait arrêté de publier ses traductions, l'ayant remplacé par un «cerveau électronique». Le patron continuait de lui envoyer du travail sur son île dans un but vaguement charitable.

Le personnage de Teddy rend bien compte de la portée et des exigences de la traduction au Québec. Son apport n'est pas à chercher du côté d'un quelconque dépaysement (après tout on ne peut guère appeler «Peanuts» une réalité étrangère), ou d'un apport d'idées nouvelles. Il se situe essentiellement au niveau de la production d'une langue normative et - pourquoi pas - d'une langue visant une certaine qualité esthétique. Éthique et esthétique se rejoignent donc dans la production d'une langue adéquate.

N'est-ce pas justement cela qui a été énoncé par l'Office de la langue française au moment de la naissance du nouvel État québécois au début des années soixante: le traducteur sera un allié dans la revalorisation de la langue. Écoutons le ton fervent de JeanMarc Léger, directeur de l'Office de la langue française en 1961. Les traducteurs, a-t-il dit, «pourront être les artisans du redressement qui doit être notre souci dominant. Ils pourront dans les divers milieux où ils vont œuvrer effacer progressivement le crime contre la nation et contre l'esprit qu'a été trop longtemps une traduction servile, pourvoyeuse d'anglicismes et instrument d'abêtissement intellectuel» (Léger 1961).

Nous voici brutalement introduits à l'héritage de la négativité que charrie la traduction au Québec. Il s'agit d'une négativité double, source à la fois de déformation linguistique et de ce qu'il a été convenu d'appeler la culture d'emprunt. Pierre Daviault (1957) a sans doute donné la formulation la plus vive et la mieux connue de ce topo au cours des années cinquante:

La pâture intellectuelle du Canadien moyen est faite, dans une très large proportion, de traduction. Nos journaux reçoivent les dépêches d'agences en anglais; en anglais aussi le texte des annonces. Nos postes de t.s.f. nous serinent de la traduction à la journée... Tous, partout et toujours, nous nous servons de textes anglais que nous traduisons ou qu'on traduit à notre intention... De la plus tendre enfance jusqu'à la fin de sa vie active, le Canadien français baigne dans une ambiance artificielle, en marge de sa culture et de toute culture.

Pour Pierre Daviault, la traduction à l'excès est génératrice d'une culture faussée, d'une non-culture. Les échos de cette dénonciation persistent encore aujourd'hui, par exemple chez le sociologue bien connu Fernand Dumont (1987: 243), qui dans un récent livre revient sur l'état de «colonisation mentale», d'exil dans des «représentations qui ne sont pas vraiment les nôtres» qui caractérise la société québécoise. Tout en soulignant l'apport souvent positif des influences extérieures, il remarque tout particulièrement «les médias de masse, la radio, la télévision, les petits journaux populaires qui déversent sur nous des messages dans une langue qui est fréquemment de traduction». Pour ces auteurs, la traduction ne contribue pas à la constitution d'une culture; elle crée une espèce de réseau parallèle et marginal.

C'est ici qu'on apprécie le revirement radical qui a caractérisé la pensée sur la traduction et son rôle social dans les années soixante-dix et quatre-vingt. Pour Jean-Claude Corbeil (1983: 31), l'un des théoriciens et des promoteurs les plus importants de la politique linguistique québécoise et en particulier de la Loi 101, on doit distinguer deux pratiques de la traduction. «Au mieux, dit-il, (la traduction est un) mode de communication à la fois interlinguistique et interculturelle» et «elle participe alors à la connaissance réciproque des cultures du monde.» «Au minimum, (cependant), et le plus souvent» (elle est) «un instrument de communication dont la fonction est de réduire l'impossibilité ou la 
difficulté de la communication entre deux locuteurs appartenant à des aires linguistiques différentes.»

Voici la traduction débarrassée de ce fardeau encombrant de la culture. Dans le schéma de Corbeil, la traduction trouve sa place dans la grande stratégie mondiale des communications; son rôle est rationalisé. La culture est un élément somme toute agréable de la communication mais elle n'y intervient pas de façon nécessaire. Denis Juhel (1983: 123) prolonge cette pensée en définissant la fonction dominante de la traduction au Québec comme étant celle de «garantir l'unilinguisme des citoyens». Nous reconnaissons ici, je pense, le même raisonnement que l'on trouve chez les promoteurs de ce que l'on appelle «l'anglais langue internationale». Il s'agit d'une conception de la pédagogie de l'anglais destinée à des hommes d'affaires et diplomates et qui conçoit la langue comme un pur outil de communication, sans que la langue soit le véhicule de valeurs propres à une culture ou à une autre (Smith, 1983).

Mais ce ne sont pas tous les participants au Colloque sur la traduction et la qualité de la langue en 1983, d'où j'ai tiré ces dernières citations, qui ont résolu ce problème de façon aussi expéditive. Jacques Maurais et Robert Dubuc, auteurs du document d'orientation, qui définissent la problématique du départ, semblent jongler avec des conceptions plus problématiques. Tout en comprenant que dans la pratique et la pédagogie de la traduction au Québec les aspects du contenu culturel de l'opération de la traduction aient été subordonnés aux valeurs expressives de la langue d'arrivée, ils se demandent si cette opération de neutralisation culturelle a été véritablement efficace. L'affrontement culturel que représente la traduction, disent-ils, dépasse le message; il réside dans l'activité ellemême. «Si cette rencontre s'opère toujours dans le même sens, il peut se développer un colonialisme culturel, par traduction interposée, qui n'est pas nécessairement à l'avantage des consommateurs de traduction» (Dubuc, Maurais 1983: 198). Contrairement à ceux qui croient que la langue peut en quelque sorte triompher de la culture, que les «valeurs de la langue de départ peuvent être remplacées» par les «valeurs de la langue d'arrivée», Dubuc et Maurais craignent que la traduction soit toujours le reflet d'un état de dépendance.

Où donc est la culture dans la langue? À défaut d'une typologie de textes, cette discussion est inévitablement vouée à un certain caractère brumeux. $\mathrm{ll}$ y a cependant une logique dans la suite de ces discours. Dubuc et Maurais semblent remettre en question la possibilité même de faire le divorce entre traduction et valeurs, du moins dans le contexte québécois où la traduction dépasse son rôle d'élément tout relatif dans l'économie des communications pour prendre une place absolue. Et de ce fait ils critiquent un certain discours qui voit dans la langue une valeur en soi. C'est le sens, me semble-t-il, de la notion d'acculturation utilisée par Jacques Flamand (1983) et Jacques Poisson (1977) pour parler d'interférences linguistiques. Pour Poisson, en particulier, porter atteinte à la langue c'est du fait même porter atteinte à la culture puisqu'elles ne font qu'un. On n'entend pas que chez les traducteurs une pareille affirmation. Au cours du débat sur le libre-échange entre le Canada et les États-Unis, on entendait dire au Québec l'argument selon lequel le Québec n'avait pas à craindre pour son autonomie culturelle face aux États-Unis parce que celle-ci était protégée, voire constituée, par la langue. On plaignait le Canada anglais, qui avait par contre tout à fait raison de craindre la menace de la culture américaine. Etait-ce à dire que les émissions de télévision américaines doublées en France et retransmises au Québec étaient garantes de cette autonomie? Toutefois, nous reconnaissons là la persistance de l'immense pouvoir symbolique de la langue au Québec.

Après ce rapide coup d'œil sur certaines positions dans un débat dont les conséquences sont pourtant réelles, on peut se demander s'il ne faut pas tout simplement reléguer aux oubliettes ces notions de langue et de culture qui sont parfois agaçantes dans 
leur manque de précision et leurs valeurs non déclarées. Dans une région discursive aussi sensible que persistante, il faut s'attendre cependant à des imprécisions et des glissements de vocabulaire. En discutant du rapport entre la traduction, la langue et la culture, nous sommes dans un domaine très chargé idéologiquement - ailleurs comme au Québec; mais en plus il s'agit ici d'un discours qui participe à la fois à un domaine de spécialistes et aux topos, aux lieux communs de la place publique. Là se situe justement son intérêt, à mon avis, parce que nous nous trouvons au cœur d'une problématique qui traverse la société tout entière.

Il faut aussi se rappeler que, n'en déplaise à Mme de Staël, la «circulation des idées» - sous forme de traduction - n'est pas nécessairement perçue comme «de tous les genres de commerce celui dont les avantages sont les plus certains». Elle l'a d'ailleurs appris à ses dépens quand sa «Lettre sur l'esprit des traductions» a déclenché des manifestations et troubles majeurs en Italie en 1816 qui ont donné lieu au mouvement romantique italien. Elle avait tout simplemement suggéré que les Italiens pourraient peut-être sortir leur littérature de son état sclérosé en traduisant massivement les littératures du Nord (Stäel 1922).

Mais en conclusion j'aimerais faire trois remarques qui pourraient contribuer à un éclaircissement autour de ces questions. D'abord, il y aurait lieu de raffiner et prolonger le modèle fonctionnel que nous proposent Corbeil et Juhel, non plus pour dire que parfois dans la traduction il y a culture et parfois pas, mais pour établir une série de vecteurs nouveaux pour rendre compte de la complexité de l'insertion sociale de la traduction. On pourrait distinguer par exemple entre les fonctions de remplacement et les fonctions incrémentielles d'un texte dans l'économie des échanges. Pourquoi fait-on appel au texte traduit et comment se greffe-t-il aux textes existants? La traduction, nous le savons, intervient tour à tour comme transmetteur de paradigmes culturels, générateur de modèles textuels, transformateur de formes du savoir ou créateur de langues. Il faut donc rendre compte de cette multiplicité de fonctions.

Ensuite, il faut bien reconnaître que culture et valeurs sont des éléments préexistant à et intervenant dans tout processus de la traduction - valeurs individuelles ou collectives qui définissent ce qui est traduit et jusqu'à un certain point comment. On pense par exemple au féminisme dans le domaine littéraire, qui a ouvert tout un nouveau champ de traductions et de retraductions, ou aux politiques linguistiques nationales ou supranationales comme celle de la $\mathrm{CEE}$, qui commande toute une industrie de la traduction. Ce postulat de base se trouve chez plusieurs théoriciens de la traduction aujourd'hui, contrairement à ce qu'on trouvait même il y a dix ans, mais de deux façons différentes. Certains définissent ces valeurs uniquement comme des contraintes et les nomment normes institutionnelles, ou systémiques, idéologies, valeurs implicites, ou bien inconscient. Le sujettraducteur est traversé par un univers discursif qu'il ou elle ne maîtrise pas. C'est ce que j'appellerais la vision rétroactive. Dans la version projective, l'on parlera plutôt de l'éthique, du projet, de projet, des valeurs sciemment invoqués lors du travail de la traduction. Ces deux perspectives se rejoignent toutefois dans la reconnaissance que la traduction s'effectue dans un contexte nécessairement baigné de valeurs.

Et, enfin, il faut distinguer la langue, comme entité symbolique, des différents discours qui la constituent. Toutes les sciences humaines depuis les années soixante ont contribué à un travail de sape au terme duquel il ne reste à peu près rien de la langue comme système homogène, comme il n'existe à peu près rien de l'équation confiante langue $=$ nation $=$ culture, ni en linguistique, ni en sociologie, ni en anthropologie, ni en philosophie. Les langues sont maintenant des mosaïques de discours, comme les nations sont des entités irrémédiablement hybrides, comme les cultures sont des ensembles pluriels de valeurs et de pratiques. La traduction participe à ce recadrement conceptuel et 
elle devient de plus en plus une figure privilégiée de la communication. Il n'est guère étonnant que le rapport entre traduction, langue et culture devienne de plus en plus complexe et fragmenté. C'est donc à l'étude des lieux très spécifiques de la traduction, mais très largement ouvert aux instances de tout ordre qui les entourent, que ces grandes questions nous renvoient.

\section{NOTE}

1. La première contribution à la question relevée par Paul Horguelin date de 1789. Il s'agit des remarques de Joseph-François Perrault, pp. 206-207, dans P. Horguelin, Anthologie de la manière de traduire, Linguatech, 1981. Voir aussi Guy Bouthillier et Jean Meynaud, Le Choc des langues au Québec, 17601960, Les Presses de l'Université du Québec, 1972.

\section{BIBLIOGRAPHIE}

BRISSET, Annie : Sociocritique de la traduction, Montréal, Éditions Préambule, à paraître.

CORBEIL, Jean-Claude (1984): «Le traducteur dans le calme ou la tourmente des communications», Traduction et qualité de langue, Éditeur officiel du Québec, pp. 130-134.

DAVIAULT, Pierre (1972): «Une culture d'emprunt», Le Choc des langues au Québec 1760-1960, Guy Bouthillier et J. Meynaud, Presses de l'Université du Québec, document no 107, pp. 649-651.

DUMONT, Fernand (1987) : Le Sort de la culture, Montréal, Éditions de 1'Hexagone.

DUBUC, Robert et Jacques MAURAIS (1984): «Document d'orientation», Traduction et qualité de la langue, Éditeur officiel du Québec, pp. 215-220.

FLAMAND, Jacques (1983): «Du bon usage de la traduction ou les risques d'aliénation culturelle dans un pays bilingue, le Canada», Dialogues et cultures, Actes du congrès de la Fédération internationale des professeurs de français.

JUHEL, Denis (1984) : «Le rôle sociolinguistique du traducteur», Traduction et qualité de langue, Éditeur officiel du Québec, pp. 120-124.

LEGGER, Jean-Marc (1962) : «L'État de la langue, miroir de la nation», Journal des traducteurs, vol. VII, $\mathrm{n}^{\circ} 2$, avril-juin, p. 39-51.

POISSON, Jacques (1977) : «La traduction, facteur d'acculturation ?», La Traduction, une profession, Actes du VIII ${ }^{e}$ congrès de la FIT, sous la direction de Paul Horguelin, Montréal.

POULIN, Jacques (1978) : Les Grandes Marées, Montréal, Leméac.

SIMON, Sherry (1989): L'Inscription sociale de la traduction au Québec, Office de la langue du Québec, coll. «Langues et société».

SMITH, Larry E. (1983): Readings in English as an International Language, Pergamon Press.

STAËL, Mme de (1922) : «De l'esprit des traductions», CEuvres complètes, Paris. 